\title{
Labyrinthine Cyst Arising in a Longstanding Cavity After a Canal Wall Down Mastoidectomy
}

\author{
Hiroaki Sato $^{*},{ }^{1}$, Koji Fukuda ${ }^{1}$, Atsuhiro Mizukawa ${ }^{1}$, Tatsuhiko Nakasato ${ }^{2}$ and Noriyuki Uesugi ${ }^{3}$ \\ ${ }^{1}$ Department of Otolaryngology, ${ }^{2}$ Department of Radiology, and ${ }^{3}$ Division of Pathology, Central Clinical Laboratory, \\ Iwate Medical University, 19-1 Uchimaru, Morioka, Iwate 020-8505, Japan
}

\begin{abstract}
Objective: Describe the clinical course and management of a labyrinthine cyst associated with a labyrinthine fistula arising in a longstanding cavity after a canal wall down mastoidectomy.

Study Design: Case report.

Setting: University Hospital, tertiary referral center.

Patients, Intervention, and Results: A 71-year-old woman who underwent left radical mastoidectomy at age 7 was referred to our clinic complaining of left ear fullness. She experienced severe vertigo for several years postoperatively, but did not complain of vertigo at her initial visit. A pale cystic mass was seen in the external auditory canal. She was completely deaf in her left ear and showed no signs of a fistula. Computed tomography and magnetic resonance imaging revealed a 12-mm mass in the superior semicircular canal and the vestibule. Removal of the cyst led to perilymph leakage through a large labyrinthine fistula, which was sealed by cortical bone chips, bone dust, and temporal fascia with fibrin glue. Histological examination revealed that the cyst wall was composed of fibrous connective tissue, contained scattered calcium deposits, and lacked an inner epithelial lining. The postoperative course was uneventful, and there has been no recurrence for 45 months.
\end{abstract}

Conclusion: To our knowledge, this is the first reported case of a labyrinthine cyst associated with a labyrinthine fistula. This delayed labyrinthine fistula is a unique complication in a longstanding cavity after a canal wall down mastoidectomy.

Keywords: Canal wall down mastoidectomy, labyrinthine cyst, canal wall down mastoidectomy, labyrinthine fistula.

Labyrinthine fistulae are one of the most frequent complications of chronic otitis media, usually caused by chronic otitis media associated with cholesteatoma [1-7]. However, these fistulae sometimes occur chronically in ears of patients who undergo a canal wall down mastoidectomy [7-9], and are called delayed labyrinthine fistulae [7]. The mechanism of this type of fistula formation is assumed to be associated not with recurrence of cholesteatoma but rather with erosion of the bony wall due to persistent inflammation of the mastoid cavity [7-10].

We present an exceptional case of a labyrinthine cyst associated with a large labyrinthine fistula, which occurred in a patient who underwent canal wall down mastoidectomy 64 years earlier, and discuss the pathogenesis and intervention. To date, this is the first reported case of a labyrinthine cyst secondary to persistent middle ear infection due to chronic otitis media.

\section{CASE REPORT}

A 71-year-old woman who had undergone left radical mastoidectomy at age 7 was referred to our clinic complaining of left ear fullness. She experienced severe vertigo for several years after her surgery, but did not

*Address correspondence to this author at the Department of Otolaryngology, Iwate Medical University, 19-1 Uchimaru, Morioka, Iwate 020-8505, Japan; Tel: +81-19-651-5111; Fax: +81-19-652-8642;

E-mail: hisato@iwate-med.ac.jp complain of vertigo currently. Left otorrhea periodically persisted after her surgery, but she had not visited a hospital until she complained of left otorrhea and ear fullness in December 2003. She had a medical history of hypertension and diabetes mellitus. Bacterial culture of the aural discharge identified 3 strains of bacteria: Serratia marcescens, Acinetobacter baumannii, and Candida sp. After an interval of 6 months, she revisited the hospital for left otorrhea. At that time, a whitish pale mass was found on the left ear, and she was referred to our clinic with suspected cholesteatoma.

The pale cystic mass approximately $10 \mathrm{~mm}$ in diameter occupied the left external auditory canal (Fig. 1). She was completely deaf in her left ear and showed no signs of a fistula. Bone algorithm computed tomography (CT) scans depicted a soft tissue mass in the left ear measuring $12 \mathrm{~mm}$ at the largest part, which was connected to the superior semicircular canal and the vestibule, and was attached to the tegmen tympani with a bone defect (Fig. 2A, B). The lateral semicircular canal was not identified; however no ossification was noted in the inner ear. Magnetic resonance imaging revealed the mass appeared to be connected to both the superior semicircular canal and the vestibule (Fig. 2C). The mass presented a $\mathrm{T} 1, \mathrm{~T} 2$ prolonged lesion, indicating a cystic lesion such as a cholesterol cyst, facial nerve neurinoma, or a labyrinthine cyst rather than a cholesteatoma.

Surgical intervention via a transmastoid approach took place on June 7, 2004. The epithelium on the facial ridge just 


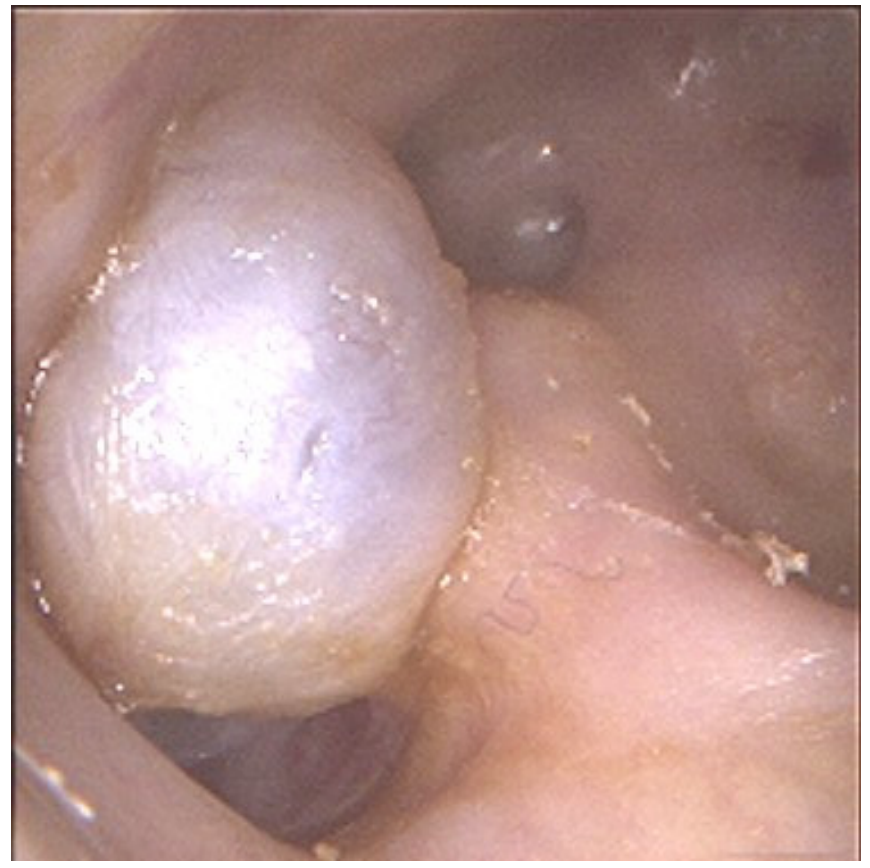

Fig. (1). Photograph of a cystic mass in the left ear.

behind the cyst was exfoliated from the underlying bone. The cyst was ruptured during this procedure, resulting in perilymph leakage. After removal of the cyst, a horizontal portion of the facial nerve was exposed on the edge of a large labyrinthine fistula between the ampullated portion of the superior semicircular canal and the vestibule, through which part of the utricular macula was seen (Fig. 3). The labyrinthine fistula was sealed with cortical bone chips, bone dust, and temporal fascia with fibrin glue. Neither nystagmus nor vertigo was observed after surgery. The postoperative course was uneventful, and the patient was discharged on day 18.

Histological examination revealed the cyst was composed of fibrous connective tissue, lacked an epithelial lining, and was covered with keratinizing squamous epithelium (Fig. 4). Inflammatory cell infiltration was scarce, but some calcification was noted in part of the cyst wall.

The patient sometimes complains of slight aural discharge, but has been free of recurrence for 45 months (Fig. 5).

\section{DISCUSSION}

Labyrinthine fistula can be caused not only by cholesteatoma, but also by chronic granulomatous otitis media without cholesteatoma $[9,10]$ or localized infection in a cavity after a canal wall down mastoidectomy [7-10]. In the present patient, there are 3 possibilities as to when the labyrinthine fistula occurred: preoperative existence secondary to cholesteatoma, intraoperative surgical trauma, and postoperative delayed labyrinthine fistula due to persistent otorrhea. The original surgical record was no longer available as the primary operation was performed 64
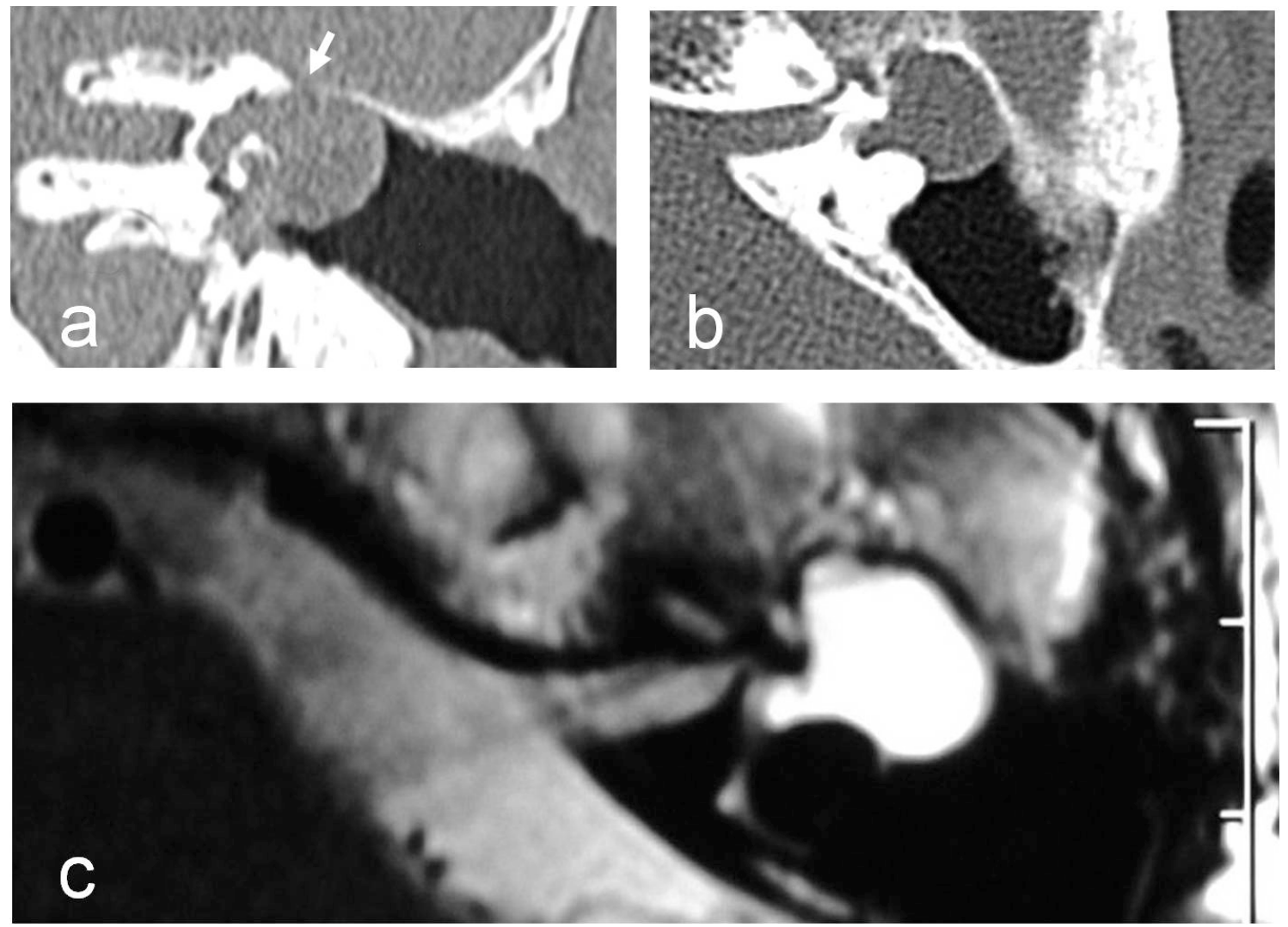

Fig. (2). Computed tomography depicts a cystic mass lacking bony partition between the mass and the vestibule on the coronal section (A) and the superior semicircular canal on the axial section (B). Bone erosion is noted in the tegmen tympani (arrow). Magnetic resonance imaging presents T1, T2 prolonged lesion, compatible with a cystic mass, that appeared to be communicating with the superior semicircular canal and the vestibule (Fig. 2C). 
years earlier. Therefore, it is impossible to tell the exact time of the labyrinthine fistula formation. However, the onset of a labyrinthine fistula seems to be due to intraoperative surgical trauma rather than preoperative or postoperative courses, as judged from the fact that severe vertigo occurred after the primary operation and persisted for several years. Postoperative labyrinthitis is another possible explanation as a cause of postoperative persistent vertigo. It is well known that the healing process for suppurative labyrinthitis results in fibrosis and new bone formation in the inner ear, that is, labyrinthitis ossificans [10]. However, CT findings, which did not show ossification in the inner ear with an absence of the lateral semicircular canal, presumably due to damage during the primary surgery, do not seem to support this mechanism.

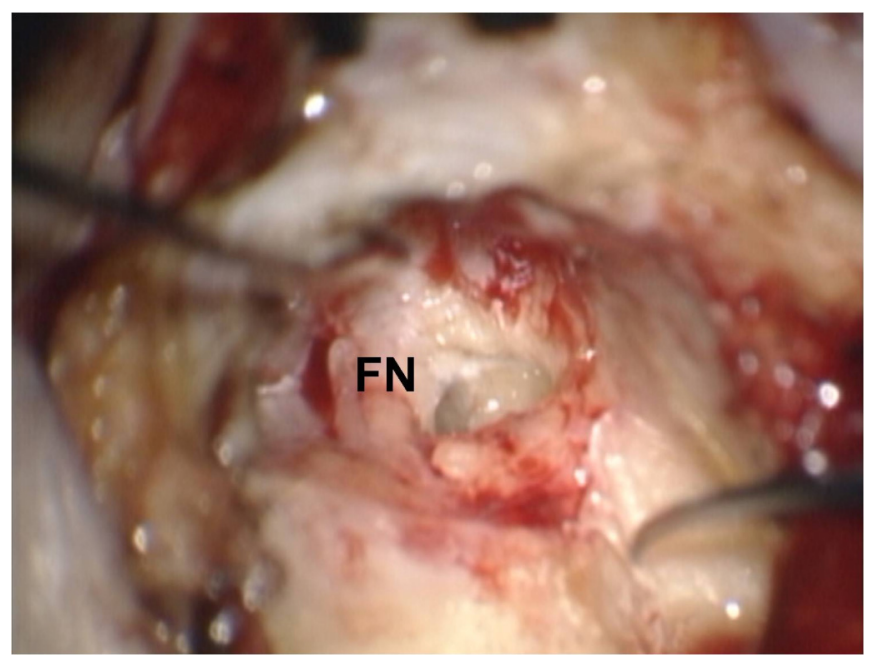

Fig. (3). Surgical view of the left ear after removal of a labyrinthine cyst. FN: facial nerve.

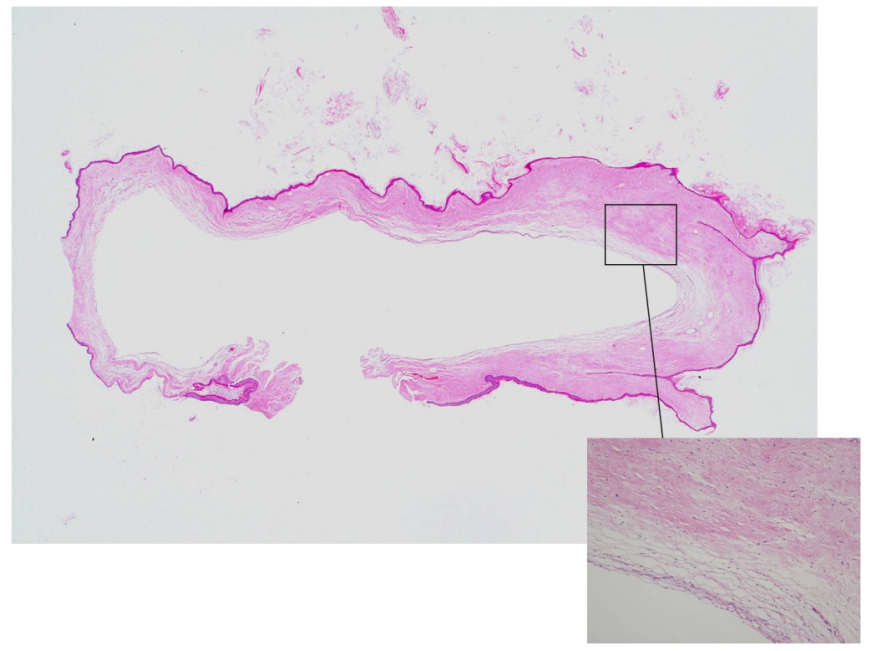

Fig. (4). Histological section of a specimen, showing a wall composed of fibrous connective tissue lacking a distinct epithelium. Original magnification $\mathrm{x} 1$, inset $\mathrm{x} 100$.

Therefore, the most plausible explanation is that an iatrogenic labyrinthine fistula, which occurred at the primary surgery 64 years earlier, gradually enlarged due to postoperative periodic infection over the long term, and resulted in the formation of a labyrinthine cyst.
Some reports have shown that labyrinthine fistulae often heal spontaneously with bony closure after removal of the cholesteatoma matrix or infection $[1,9,11]$. However, labyrinthine fistulae sometimes occur in a cavity after a canal wall down mastoidectomy due to persistent infection, without recurrence of cholesteatoma [7-9]. These observations indicate that control of infection in the middle ear is essential for prevention of delayed labyrinthine fistulae or promotion of spontaneous closure of labyrinthine fistulae. Currently, the present patient has been free of recurrence for 45 months after surgical closure using cortical bone chips, bone dust, and temporal fascia with fibrin glue. We selected closure of a labyrinthine fistula instead of total middle ear obliteration as the latter may increase the risk of inability of controlling the infection when obliterated at one stage. However, the patient already lost her hearing on the affected side at initial visit and she sometimes complains of slight aural discharge after surgery. Taking these into consideration, total obliteration of the middle ear cavity would be preferable for prevention of possible recurrence of the cyst in future.

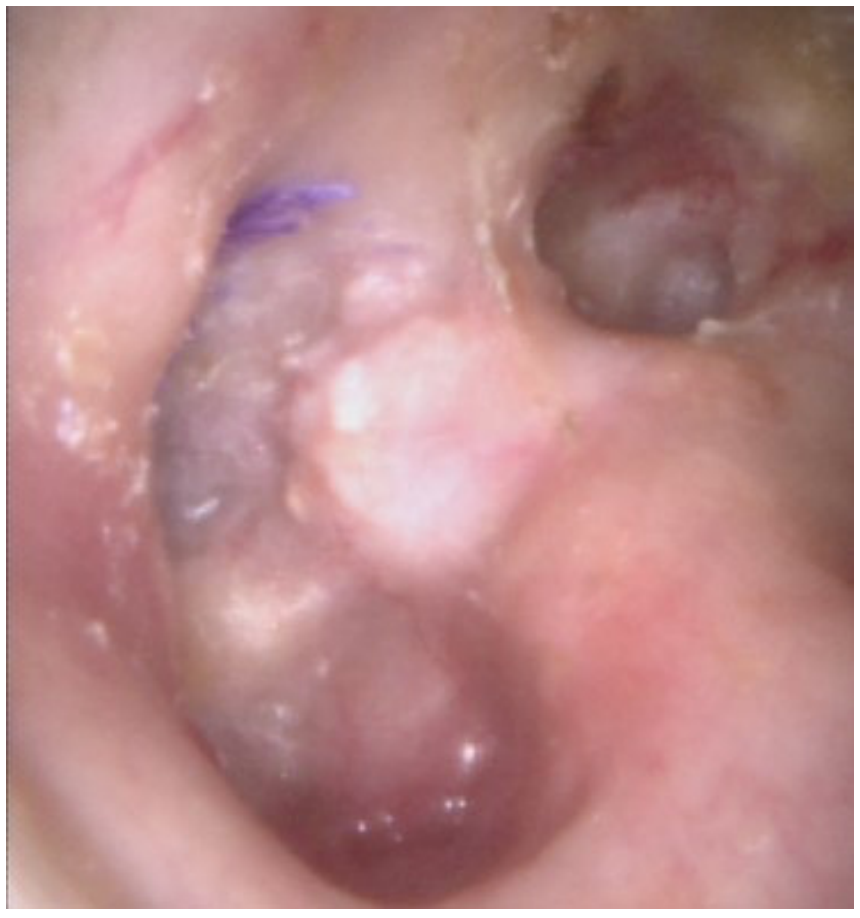

Fig. (5). Postoperative finding of the left ear.

To our knowledge, there have been no reports of a cyst in the middle ear connecting to the labyrinth except for a congenital cyst of the oval window, in which pneumococcal meningitis had recurred due to spontaneous cerebrospinal fluid leakage [12].

\section{CONCLUSION}

The present case represents the first report of a labyrinthine cyst associated with labyrinthine fistula in a longstanding cavity after a canal wall down mastoidectomy. Persistent inflammation in the cavity after the canal wall down mastoidectomy may cause not only delayed labyrinthine fistulae but also a labyrinthine cyst. 


\section{REFERENCES}

[1] Sanna M, Zini C, Bacciu R, Scandellari R, Delogu RS, Jemmi G. Management of the labyrinthine fistula in cholesteatoma surgery. ORL J Otorhinolaryngol Relat Spec 1984; 46: 165-72.

[2] Magliulo G, Terranova G, Varacalli S, et al. Labyrinthine fistula as a complication of cholesteatoma. Am J Otol 1997; 18: 697-701.

[3] Gersdorff MCH, Nouwen J, Decat M, et al. Labyrinthine fistula after cholesteatomatous chronic otitis media. Am J Otol 2000; 21: 32-5.

[4] Soda-Merphy A, Betancourt-Suarez MA. Surgical treatment of labyrinthine fistula caused by choesteatoma. Otolaryngol Head Neck Surg 2000; 122: 739-42.

[5] Ahmad I, East DM. Hearing preservation in patients with labyrinthine fistulae. Rev Laryngol Otol Rhinol 2002; 123: 49-52.

[6] Grewal DS, Hathiram BT, Dwivedi A, et al. Labyrinthine fistula: a complication of chronic suppurative otitis media. J Laryngol Otol 2003; 117: 353-7.
[7] Portmann D, Ferreira DR. Delayed labyrinthine fistula in canal wall down mastoidectomy. Rev Laryngol Otol Rhinol 2003; 124 : 265-8.

[8] Hakuba N, Hato N, Shinomori Y, et al. Labyrinthine fistula as a late complication of middle ear surgery using the canal wall down technique. Otol Neurotol 2002; 23: 832-5.

[9] Jang CH, Merchant SN. Histopathology of labyrinthine fistulae in chronic otitis media with clinical implications. Am J Otol 1997; 18: $15-25$.

[10] Schucknecht HF. Pathology of the ear. Philadelphia: Lea Febiger, 1993; pp. 211-8.

[11] McCabe BF. Labyrinthine fistula in chronic mastoiditis. Ann Otol Rhinol Laryngol 1984; 93 (supp1112): 138-41.

[12] Lahovary A: Kongenitale Zyste im ovalen Fenster. HNO 1978; 26 : 419-20. 